\title{
A mathematical model for the capillary endothelial cell-extracellular matrix interactions in wound-healing angiogenesis
}

\author{
LUKE OLSEN \\ Centre for Mathematical Biology, Mathematical Institute, \\ 24-29 St Giles', Oxford OX1 3LB, UK \\ JONATHAN A. SHERRATT $\dagger$ \\ Nonlinear Systems Laboratory, Mathematics Institute, \\ University of Warwick, Coventry, CV4 7AL, UK \\ PHILIP K. MAINI \\ Centre for Mathematical Biology, Mathematical Institute, \\ 24-29 St Giles', Oxford OXI 3LB, UK \\ FRANK ARNOLD \\ Institute for Wound Healing, Department of Dermatology, \\ Churchill Hospital, Oxford OX3 7LJ, UK
}

[Received 21 September 1996 and in revised form 27 February 1997]

\begin{abstract}
Angiogenesis, the process by which new blood capillaries grow into a tissue from surrounding parent vessels, is a key event in dermal wound healing, malignant-tumour growth, and other pathologic conditions. In wound healing, new capillaries deliver vital metabolites such as amino acids and oxygen to the cells in the wound which are involved in a complex sequence of repair processes. The key cellular constituents of these new capillaries are endothelial cells: their interactions with soluble biochemical and insoluble extracellular matrix (ECM) proteins have been well documented recently, although the biological mechanisms underlying wound-healing angiogenesis are incompletely understood. Considerable recent research, including some continuum mathematical models, have focused on the interactions between endothelial cells and soluble regulators (such as growth factors). In this work, a similar modelling framework is used to investigate the roles of the insoluble ECM substrate, of which collagen is the predominant macromolecular protein. Our model consists of a partial differential equation for the endothelial-cell density (as a function of position and time) coupled to an ordinary differential equation for the ECM density. The ECM is assumed to regulate cell movement (both random and directed) and proliferation, whereas the cells synthesize and degrade the ECM. Analysis and numerical solutions of these equations highlights the roles of these processes in wound-healing angiogenesis. A nonstandard approximation analysis yields insight into the travelling-wave structure of the system. The model is extended to two spatial dimensions (parallel and perpendicular to the plane of the skin), for which numerical simulations are presented. The model predicts that ECM-mediated random motility and cell proliferation are key processes which drive angiogenesis and that the details of the functional dependence of these processes on the ECM density, together with the rate of ECM remodelling, determine the qualitative nature of the angiogenic response. These predictions are experimentally testable, and they may lead towards a greater understanding of the biological mechanisms involved in wound-healing angiogenesis.
\end{abstract}

Keywords: wound healing; angiogenesis; travelling waves.

$\dagger$ Author to whom all correspondence should be addressed. E-mail: jas@maths.warwick.ac.uk. 


\section{Introduction}

Cells of all living organisms require a variety of biochemical nutrients in order to function normally and to respond to environmental signals. In tissues larger than a critical size, diffusion of these substances is an inadequate supply method and a circulatory system is needed. Important examples of this phenomenon include solid-tumour and healing-wound tissue, both of which are restricted to a linear dimension of a few millimetres until they are invaded by blood capillaries (Alberts et al., 1989; Clark, 1993; Folkman \& Shing, 1992).

Angiogenesis, defined as the ingrowth of a capillary network from pre-existing vessels in the surrounding tissue, may therefore be either beneficial (as in wound healing) or detrimental (as in tumour growth). Thus, increasing understanding of the biological mechanisms involved in angiogenesis and of how these processes are regulated are important steps in improving clinical treatments for malignant tumours and abnormally healing wounds.

In this paper, we propose a continuum mathematical model for wound-healing angiogenesis which can be used to investigate the regulatory roles of the solid-state extracellular matrix (ECM) in the behaviour of blood capillaries.

\subsection{Biological background}

Mammalian skin cells obtain vital metabolites such as oxygen from an extensive capillary network throughout the dermis, which is connected to larger arteries and veins in the subdermal layers (Mast, 1992). Consequently, the dermal response to injury must not only restore this capillary network but must also provide extra nutrients for the increased number of metabolically active cells involved in repair (Arnold \& West, 1992). Angiogenesis, which typically parallels fibroplasia and wound contraction (from a couple of days to a couple of weeks post-wounding), is therefore a key event in the healing of full-thickness dermal excisional skin wounds (Clark, 1993).

In normal unwounded skin, blood capillaries constitute an essentially quiescent network of endothelial cells which are organized into tubular structures. Laminae of insoluble extracellular matrix (ECM) proteins known as the basement membrane coat the exterior of blood capillaries and are believed to maintain endothelial stability, impermeability, and resistance against the hydrostatic pressure of the blood flow within vessels (Ryan, 1989).

An early event in wound angiogenesis is the proteolytic degradation of the basementmembrane ECM of vessels around the wound margins in response to wound-induced angiogenic stimuli. These signals probably arise from the enzymatic cascades which characterize the early, inflammatory phase of repair (Clark, 1993), although wound-induced vessel dilation may also be significant (Whalen \& Zetter, 1992).

At vessel locations where the basement membrane has been degraded, capillary endothelial cells become activated, assuming a migratory and proliferative phenotype (Folkman \& Shing, 1992). A few cells initially migrate out from the capillary wall towards the wound site, possibly mediated by basement-membrane fragments, by exposure to the connectivetissue ECM, or in response to (diffusible) angiogenic factors in the wound (Iruela-Arispe \& Sage, 1993; Mardri \& Pratt, 1988). 
Immediately behind the advancing endothelial bud, cells proliferate and differentiate into tubular morphologies, thereby forming a new capillary (Ingber \& Folkman, 1989a). Cells further behind the leading tip also begin to synthesize new ECM substances-notably collagen, the predominant protein of normal dermis.

Blood flow is restored when two new capillary tips join to form a closed loop. Secondary buds may sprout from these new vessels via similar mechanisms of endothelial-cell activation, migration, and proliferation. In this way, the wound tissue becomes endowed with a dense network of fine blood capillaries which, in time, develop into mature, quiescent vessels with increasing diameters and ECM (especially basement membrane) deposition. The total number of vessels declines from its peak around a week post-wounding, although the mechanisms of vascular maturation and regression are unclear (Dyson et al., 1992; Whalen \& Zetter, 1992).

The factors which regulate wound angiogenesis have attracted considerable recent research (for reviews see Arnold \& West, 1992; Folkman \& Shing, 1992; Whalen \& Zetter, 1992); they may be broadly subdivided into the following two categories:

(i) soluble signals, cytokines (for example, fibroblast growth factor and platelet-derived growth factor), metabolic gradients (for example, in oxygen and lactate), and proteases (for example, collagenase, plasminogen activator, and urokinase).

(ii) insoluble signals, ECM substrate (for example, fibrillar collagen and fibronectin) and physical forces (the morphology and behaviour of capillary endothelial cells is influenced by mechanical stresses in vitro).

Although many factors have been shown to affect endothelial-cell function in culture, their roles in vivo are difficult to assess experimentally, because of the multiple direct and indirect interactions between individual components of the overall wound-healing process.

Soluble (or diffusible) angiogenic regulators have been more widely studied. Gradients of metabolic factors (such as oxygen and lactate) across a wound are believed to regulate endothelial-cell activity, whilst growth factors may stimulate cell movement (via chemotaxis), proliferation, and ECM synthesis (Drucker et al., 1996; Jonsson et al., 1991; Knighton et al., 1982). Proteolytic enzymes also mediate cell-ECM interactions such as attachment, detachment, and force transduction between the intracellular cytoskeleton and the ECM (Ingber, 1993; Ryan, 1989).

The fibrillar ECM is undoubtedly an important local regulator of angiogenesis. For example, the concentrations of collagen and/or fibronectin substrates may determine whether cultured capillary endothelial cells migrate, proliferate, or differentiate into tubular, capillary-like structures (Ingber \& Folkman, 1989a: Ryan, 1989). These substrates have also been demonstrated to promote cell movement by haptotaxis, haptokinesis, and contact guidance in vitro (Dickinson \& Tranquillo, 1993; McCarthy et al., 1988). In addition, inhibition of ECM remodelling modulates angiogenesis (Ingber \& Folkman, 1988). The further roles of the ECM in angiogenesis include the mediation of growth-factor-driven endothelial-cell behaviour (Iruela-Arispe \& Sage, 1993; Sato et al., 1993) and support for the mechanical effects of cell-ECM interactions (for example, cell traction) which may orchestrate wound healing via directional cues and ECM-fibre alignment (Clark, 1993; Ryan, 1989; Stopak \& Harris, 1982; Vernon \& Sage, 1995). 


\subsection{Mathematical-modelling background}

Recently, Chaplain and colleagues have proposed continuum mathematical models for the (diffusible) chemical regulation of tumour angiogenesis (Byrne \& Chaplain, 1995; Chaplain \& Stuart, 1991a,b). Specifically, a generic tumour angiogenesis factor is secreted by the tumour, diffuses freely in the tissue, decays naturally, and is metabolized by capillary endothelial cells. In addition, these cells proliferate and migrate chemotactically in response to the chemical. These dynamics are modelled using two coupled nonlinear partial differential equations, governing: (i) the chemical concentration, and (ii) the density of capillary-tip endothelial cells. Variants of the basic model framework account for capillary-loop formation and for the dynamics of intact blood vessels behind the leading capillary tips.

Given the recognized biological similarities between tumour and wound angiogenesis (Chaplain \& Byrne, 1996; Folkman \& Shing, 1992), the above model framework may also be relevant to wound healing, wherein growth factors released at the wound site by inflammatory cells perform analogous roles to the tumour angiogenesis factor. Some authors have now considered this application of the 'generalized' chemically regulated angiogenesis model (Chaplain \& Byrne, 1996; Pettet et al., 1996).

\section{A new mathematical model}

Previous mathematical models have neglected the ECM involvement in angiogenesis. In this work, we develop and analyse a new model, based on a framework similar to that outlined above, to investigate how interactions between capillary endothelial cells and the solid-state ECM substrate may regulate wound angiogenesis. Our model has a relatively simple form, and represents a first attempt to simulate the ECM-endothelial-cell interaction during wound angiogenesis. Potential extensions to more realistic representations are discussed in Section 6.

\subsection{Framework and variables}

The independent variables are as follows.

(i) Time, $t$, measured from the onset of the proliferative phase of repair when angiogenesis commences-usually 2 or 3 days post-wounding (Clark, 1993).

(ii) Spatial position, $\underline{r}$; we neglect the effects of wound depth and consider the onedimensional (1D) geometry in the plane of skin suggested by a long rectangular (or 'linear') wound, where $-L \leqslant x \leqslant L$ defines the wound space, with the wound centre at $x=0$. The unwounded dermis occupies the region $|x|>L$.

The dependent variables must, at least, represent the blood capillaries and the insoluble ECM. In order to simulate their interactions within the context of a healing wound rather than in the immediate vicinity of individual capillaries, these quantities are defined as local spatial continuum averages:

(i) capillary endothelial-cell density, $n(x, t)$; note that this term does not account for the capillary orientation, which would require a more complicated vector-field representation (which is beyond the scope of this paper); 
(ii) solid-state fibrillar ECM density, $m(x, t)$, whose properties are modelled on those of collagen, the predominant structural protein of the dermis.

\subsection{One-dimensional model equations}

As detailed above, we consider the following, fundamental biological interactions between (capillary endothelial) cells and the (collagenous) ECM that are known to occur during wound angiogenesis:

Cells, unbiased migration mediated by the ECM (haptokinesis), biased migration up ECM density gradients (haptotaxis), and mitotic division (proliferation);

$E C M$, production (biosynthesis) and degradation (proteolysis) by cells.

Thus, the ECM regulates cell movement and proliferation-experimentally, these effects are stimulatory at low ECM densities and inhibitory at relatively high densities (Dickinson \& Tranquillo, 1993; Nusgens et al., 1984; Weinberg \& Bell, 1985; Yoshizato et al., 1985).

The above considerations suggest the following model equations, which govern the cell and ECM densities:

$$
\begin{aligned}
& \frac{\partial n}{\partial t}+\overbrace{\frac{\partial}{\partial x}\left[C(m) n \frac{\partial m}{\partial x}-D(m) \frac{\partial n}{\partial x}\right]}^{\text {haptotaxis and haptokinesis }}=\overbrace{[A(m)-B n] n}^{\text {proliferation }} \equiv f(n, m), \\
& \frac{\partial m}{\partial t}=\overbrace{(P-Q m) n}^{\substack{\text { production and } \\
\text { degradation }}} \equiv g(n, m) \text {. }
\end{aligned}
$$

The model parameters are all non-negative and the cellular functions also satisfy the following particular constraints.

(i) The haptotactic coefficient, $C(m)=C_{0}\left(K_{C}+m\right)^{-2}$, is derived from kinetic analysis of a model mechanism for the cell-surface-receptor-extracellular-ligand binding dynamics (Sherratt, 1994); $C_{0} \geqslant 0$ and $K_{C}>0$.

(ii) The haptokinetic coefficient, $D(m)=D_{0} m\left(K_{D}^{2}+m^{2}\right)^{-1}$, is suggested by specific experimental data (Dickinson \& Tranquillo, 1993) and by the intuitive arguments that cells cannot move in the absence of a substrate for attachment (so $D=0$ when $m=0$ ) and that cell movement is restricted when the ECM becomes sufficiently dense (so $D \rightarrow 0$ as $m \rightarrow \infty) ; D_{0} \geqslant 0$ and $K_{D}>0$.

(iii) The cell proliferation rate, $A(m)=A_{0} m\left(K_{A}^{2}+m^{2}\right)^{-1}$, is also motivated by experimental findings (Weinberg \& Bell, 1985; Yoshizato et al., 1985) and by biological arguments similar to those for the haptokinetic coefficient (above); $A_{0} \geqslant 0$ and $K_{A}>0$.

\subsection{End conditions}

The following boundary conditions are associated with equations (1) and (2). By symmetry, solutions need only be defined on the semi-infinite domain $x \geqslant 0$ subject to zero flux at the wound centre; that is, $C(m) n \partial m / \partial x=D(m) \partial n / \partial x$ at $x=0$. Also, the cell and ECM 
variables remain at their normal dermal values far away from the wound, so $n \rightarrow n_{0}$ and $m \rightarrow m_{0}$ (say) as $x \rightarrow \infty$.

Initially, there are no endothelial cells and a low level of ECM (due to the early deposition of provisional matrix) inside the wound, so $n=0$ and $m=m_{\text {init }}$ (say) at $t=0$ for $0 \leqslant x \leqslant L$, with the unwounded values $n=n_{0}$ and $m=m_{0}$ outside the wound $(x>L)$. Note that $0<m_{\text {init }}<m_{0}$.

\subsection{Existence and stability of equilibria}

Spatially uniform equilibria of equations (1) and (2) are given by the solutions of $f(n, m)=$ $g(n, m)=0$. Their local stability is deduced from linear analysis, and the global stability is investigated using standard phase-plane analysis of the spatially independent system. The following results are obtained.

(i) A continuum of unstable 'acellular' states, in which $n=0$ and $m$ is unspecified.

(ii) A globally stable state, in which $n=A(m) / B$ and $m=P / Q$. These values are positive, and equated to $n_{0}$ and $m_{0}$, respectively, so that this 'dermal' steady state represents normal, unwounded tissue.

These results imply that the initial 'wound state' evolves towards the same dermal state as existed before injury, which is indicative of wound healing. The spatiotemporal nature of these dynamics and the roles of the biological processes in the model will be investigated in the remainder of the paper.

\subsection{Nondimensionalization}

The variables and parameters in equations (1) and (2) and their associated end conditions are transformed into dimensionless quantities via the following scalings:

$$
\begin{gathered}
\tilde{t}=\frac{t}{T}, \quad \tilde{x}=\frac{x}{L}, \quad \tilde{n}=\frac{n}{n_{0}}, \quad \tilde{m}=\frac{m}{m_{0}}, \quad \chi(\tilde{m})=\frac{C(m) T}{L^{2}}, \quad \tilde{D}(\tilde{m})=\frac{D(m) T}{L^{2}}, \\
\alpha(\tilde{m})=A(m) T, \quad \beta=B n_{0} T, \quad \epsilon=Q n_{0} T, \quad \widetilde{m_{\text {init }}}=\frac{m_{\text {init }}}{m_{0}} .
\end{gathered}
$$

The time scale, $T$, is defined as 1 day. Equations ( 1 ) and (2) thus become (omitting the tildes for notational clarity)

$$
\begin{aligned}
\frac{\partial n}{\partial t}+\frac{\partial}{\partial x}\left(\chi(m) n \frac{\partial m}{\partial x}-D(m) \frac{\partial n}{\partial x}\right) & =[\alpha(m)-\beta n] n \equiv f(n, m), \\
\frac{\partial m}{\partial t}=\epsilon(1-m) n & \equiv g(n, m) .
\end{aligned}
$$

Note that the dimensionless unwounded dermal equilibrium is $(n, m)=(1,1)$ since $\beta=$ $\alpha(1)$.

Next, numerical simulations of the dimensionless model are presented and the mathematical structure of these solutions are analysed. 

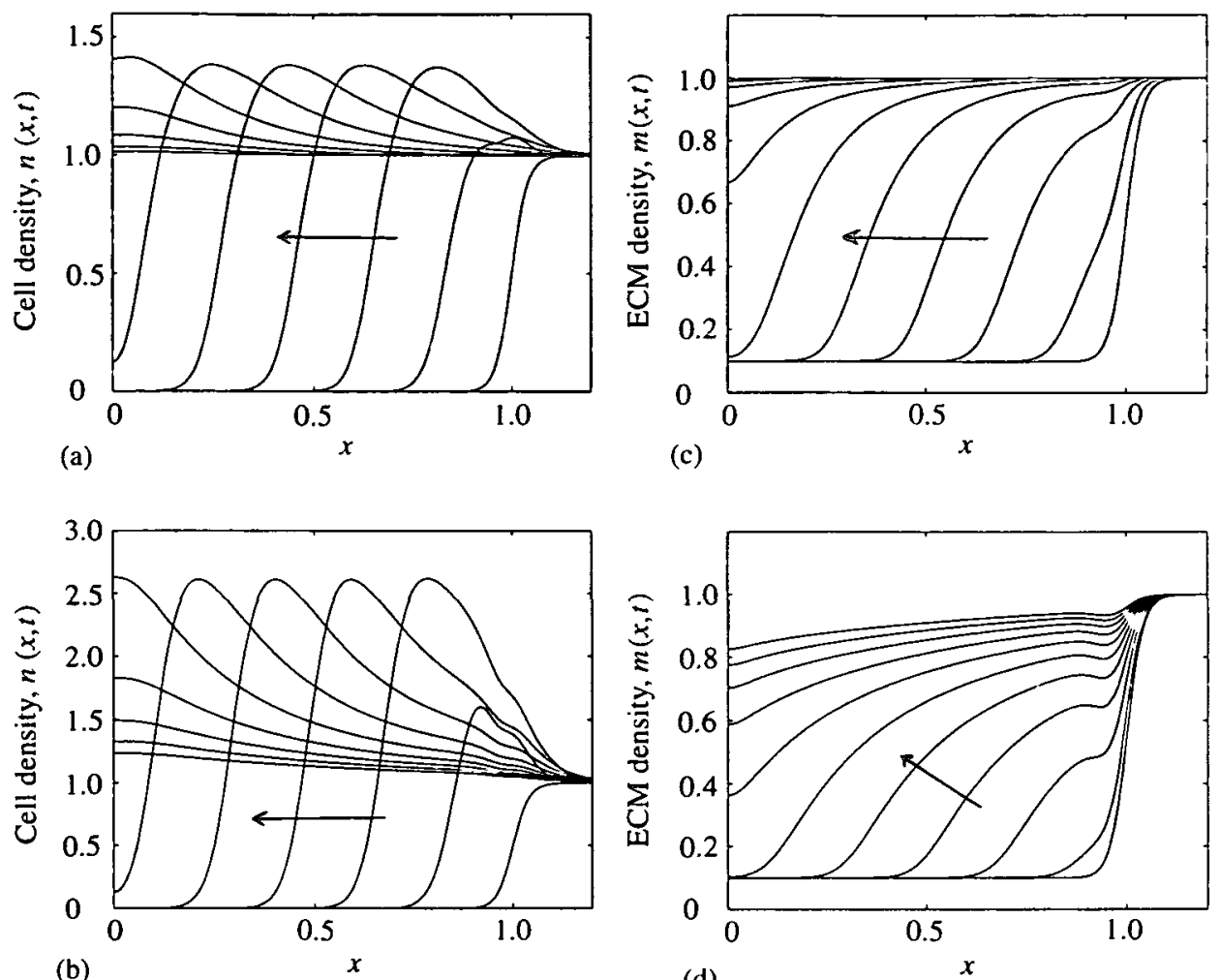

FIG. 1. A numerical simulation of vascular growth into a wound. The wavefronts $(a, b)$ of the endothelial-cell density and (c,d) of the ECM density move into the wound in parallel. We plot the solutions at time $t=0$ and at ten successive unit intervals. The wound space is assumed to be one dimensional, so that we are modelling the healing of an incisional wound. The point $x=1$ corresponds to the initial wound edge, and $x=0$ corresponds to the wound centre. The model equations (3) and (4) were solved numerically using methods described in the Appendix. The end conditions are given in the text. The parameter values were: $(a, c) D_{0}=0.005, \kappa_{D}=0.5$, $\chi_{0}=0.001, \kappa_{\chi}=0.5, \alpha_{0}=1.01, \kappa_{\alpha}=0.1, \beta=1, \epsilon=1$, and $m_{\text {intt }}=0.1$; (b,d) the same except that $\epsilon=0.2$, representing slower ECM kinetics. The finte-difference approximation mesh sizes, $\Delta x=\Delta t=0.005$, are considerably smaller than the bounds for numerical stability, in order to yield high accuracy. The arrows indicate the direction of the wavelike propagation of the cells and ECM towards the wound centre.

\section{Numerical simulations}

Numerical solutions of equations (3) and (4) with the nondimensional end conditions are obtained using a semi-implicit finite-difference scheme (see the Appendix for details).

A typical numerical simulation of the model is presented in Fig. 1, which shows the endothelial cell density, $n$, and the ECM density, $m$, propagating into the wound space $(0 \leqslant x \leqslant 1)$ in a wavelike form. The key features of these dynamics are:

(i) the cell and ECM profiles appear to evolve with approximately constant speed and form, after an initial transient and before the profiles reach the wound centre $(x=0)$;

(ii) the cell density increases above its normal dermal value (unity) in the wavefront before gradually returning to its unwounded level, whereas the ECM profile is monotonically increasing; 
(iii) the gradients of the profiles are steeper in the wavefront than in the waveback.

These phenomena simulate some important qualitative aspects of wound angiogenesis in vivo; in particular, endothelial-cell number increases sharply prior to a gradual regression which parallels ECM accumulation. In Section 8, we will discuss in vitro procedures which could be used to replicate these results in detail.

Figure 1 also illustrates that these features are more pronounced, and more realistic, when the ECM kinetics rate parameter $\epsilon$ is smaller. In wound healing, ECM remodelling occurs on a slower time scale than cellular proliferation (Clark, 1988), implying that this parameter should be relatively small in magnitude (Olsen et al., 1995).

\section{Travelling-wave analysis}

The forms of the numerical solutions of the model suggest that profiles with constant speed and form may evolve from the initial conditions. If so, then the dependence of these attributes on the biological parameters in the model are of central importance in gaining insight into ECM regulation of wound angiogenesis. Travelling-wave solutions have been observed in other nonlinear parabolic partial-differential-equation models in cellular biology and wound healing (Byrne \& Chaplain, 1995; Dale et al., 1994; Olsen et al., 1996; Sherratt et al., 1992).

\subsection{Existence of travelling-wave solutions}

We begin our analysis by considering the possible speeds for which the model equations have wave solutions of the form observed in simulations of wound healing (see Fig. 1). This is an essential precursor to determining an expression for the wave speed in terms of the model parameters. Such an expression is discussed further in Section 6, and it corresponds biologically to the predicted speed of vascular ingrowth.

Travelling-wave solutions are investigated first by the variable transformations

$$
z=x+c t, \quad N(z)=n(x, t), \quad M(z)=m(x, t),
$$

where $c>0$ is the constant wave speed. Substituting into equations (3) and (4) yields the following system of ordinary differential equations:

$$
\begin{aligned}
c N^{\prime}+\left[\chi(M) N M^{\prime}-D(M) N^{\prime}\right]^{\prime} & =f(N, M)=[\alpha(M)-N] N, \\
c M^{\prime}=g(N, M) & =\epsilon(1-M) N,
\end{aligned}
$$

where the primes denote differentiation with respect to $z$.

If travelling-wave solutions exist, then a heteroclinic trajectory must exist from the 'wounded equilibrium' $\left(0,0, m_{\text {init }}\right)$ to the 'dermal equilibrium' $(1,0,1)$ in $\left(N, N^{\prime}, M\right)$ space, lying wholly in the biologically realistic region $N \geqslant 0, M \geqslant 0$. The existence of such a trajectory necessitates at least one unstable eigenvalue at $\left(0,0, m_{\mathrm{init}}\right)$, and at least one stable eigenvalue at $(1,0,1)$.

Standard linear-stability analysis of equations (5) and (6) in $\left(N, N^{\prime}, M\right)$-space (see, for example, Murray, 1989; Edelstein-Keshet, 1988$)$ reveals that the eigenvalues at $\left(0,0, m_{\text {init }}\right)$ 
are

$$
0, \quad \frac{c \pm \sqrt{c^{2}-4 D\left(m_{\text {1nit }}\right) \alpha\left(m_{\text {init }}\right)}}{2 D\left(m_{1 \mathrm{mit}}\right)} .
$$

Since $c$ and $D$ are positive at least one eigenvalue is positive, as required. If some eigenvalues are complex, however, then locally oscillatory solutions imply that $N(z)<0$ for some $z$, which is biologically inadmissible. Thus, the eigenvalues must be real, giving the following minimum wave-speed condition:

$$
c \geqslant c_{\min }=2 \sqrt{D\left(m_{1 \mathrm{nit}}\right) \alpha\left(m_{\mathrm{1n \textrm {I }}}\right)}
$$

At $(1,0,1)$, there is one real positive and two real negative eigenvalues for all values of $c$, which does not impose further constraints on the wave speed. Thus, travelling-wave solutions may exist for any wave speed greater than $c_{\min }$ given in (7). In Section 6, we assess whether the theoretical minimum wave speed, $c_{\mathrm{mmn}}$, is observed in numerical simulations. We also investigate the effects of haptotaxis (coefficient $\chi$ ); the nonlinear haptotactic term is absent from the expression for $c_{\min }$ because condition (7) is derived from linear analysis (above).

\subsection{Perturbation approximations}

Having established the possible existence of travelling-wave solutions with a speed bounded below by $c_{\min }$, we now use perturbation theory to study the form of the wave solutions. The aim of this subsection is to determine the way in which features such as the peak in the endothelial-cell density and the slope of the ECM wave depend on the model parameters. Mathematically, our approach is motivated by the dependence of the waveforms on the small parameter $\epsilon$ in the numerical simulations, as illustrated in Fig. 1. Recall that the size of $\epsilon$ reflects slow rates of ECM synthesis and degradation.

The leading order $(\epsilon=0)$ approximation to equations (6) and (7) implies that $M^{\prime}=0$, and hence that $M(z)$ is constant. This fails to satisfy the boundary conditions that $M \rightarrow$ $m_{\text {init }}$ as $z \rightarrow-\infty$ and that $M \rightarrow 1$ as $z \rightarrow \infty$, however, so the perturbation $\epsilon>0$ is singular.

Figure 1 (and similar numerical simulations) reveals that as $\epsilon$ decreases the domain of influence, which is defined formally in the legend to Fig. 2, of the dynamics increases behind the wave. This suggests that in the waveback, $z$ can be rescaled so that the domain of influence is invariant in the $\xi$-domain, where $\xi=\Omega(\epsilon) z$ and $\Omega$ is a positive increasing function of $\epsilon$ which is to be determined. By quantifying how the domain of influence depends on $\epsilon$ in numerical simulations, as illustrated in Fig. 2 we can deduce that $\Omega \sim \epsilon$, so that an appropriate transformation in the waveback is $\xi=\epsilon z$; that is, the width of the $E C M$ wavefront is inversely proportional to the rate of ECM dynamics. We will now use this rescaling to determine the qualitative form of the waves, by considering the solution separately in the wavefront and the waveback, and then matching these two solution parts using standard techniques.

4.2.1 Wavefront approximation. In the wavefront, the nontransformed equation (7) implies that $M(z) \equiv m_{\text {init }}$ to lowest order, using the boundary condition far ahead of the wave. 


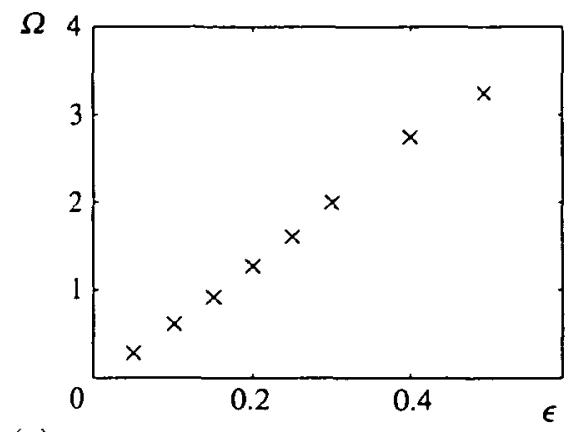

(a)

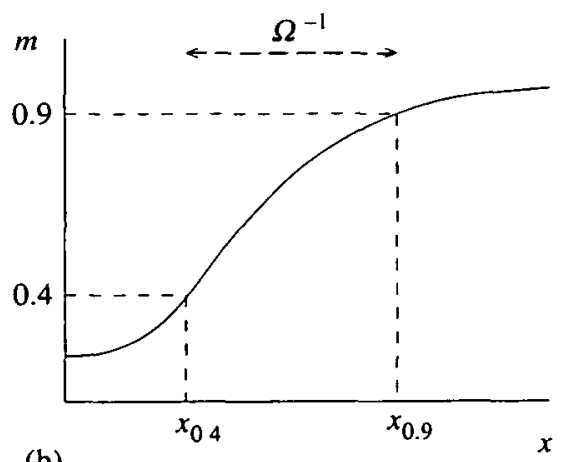

(b)

FIG. 2. (a) Numerical measurements of $\Omega$ for different values of $\epsilon$. The parameter $\epsilon$ reflects the rate of $E C M$ kinetics (synthesis and degradation by cells), and is consequently small, while $\Omega^{-1}$ represents the width of the wave of the ECM density $m$ associated with vascular ingrowth. For a fixed $\epsilon$, the spatial domain of influence, $\Omega^{-1}$, is defined as the difference $x_{09}-x_{04}$, as illustrated in (b), where $x_{s}$ is determined by $m\left(x_{s}, t\right)=s$, at some given time $t$ which remains fixed throughout these simulations. The profiles of $n$ and $m$ given in Fig. 1 confirm that values of $m$ between 0.4 and 0.9 lie wholly within the waveback. By plotting $\Omega$ against $\epsilon$, it is evident that $\Omega$ and $\epsilon$ are approximately proportional. Note that the protracted spatial domain $-10 \leqslant x \leqslant 1.2$ was used to accurately calculate $\Omega$ and that the wave speed was independent of $\epsilon$ in these numerical experiments, so that measuring $\Omega$ in terms of $x$ instead of $z$ is valid ( $t$ was kept fixed throughout). The calculation presented in this figure motıvated our choice of scaling in the perturbation analysis.

Equation (6) then simplifies to

$$
c N^{\prime}=D\left(m_{\text {init }}\right) N^{\prime \prime}+\left[\alpha\left(m_{\text {nit }}\right)-\beta N\right] N,
$$

which is the Fisher equation (Fisher, 1937), whose travelling-wave behaviour has been extremely well studied (Murray, 1989).

4.2.2 Waveback approximation. In the waveback, the equations are expressed in terms of $\xi$ via the transformation $\xi=\epsilon z$ (as discussed above), leading to

$$
N(\xi)=\alpha[M(\xi)] / \beta
$$

from equation (6) and to

$$
\frac{\mathrm{d} M}{\mathrm{~d} \xi}=(1-M) \alpha(M) / \beta
$$

from equation (7). Using the functional form $\alpha(M)=\alpha_{0} M /\left(k_{\alpha}^{2}+M^{2}\right)$, this ordinary differential equation can be solved implicitly to give

$$
\kappa_{\alpha}^{2} \ln M-\left(\kappa_{\alpha}^{2}+1\right) \ln (1-M)-M=\alpha_{0}\left(\xi-\xi_{0}\right) / \beta
$$

where $\xi_{0}$ is an arbitrary constant of integration to be determined by matching the solutions in the two portions of the domain (see below). Note that the boundary conditions $M \rightarrow 1$ and $N \rightarrow 1$ as $\xi \rightarrow \infty$ are satisfied. 


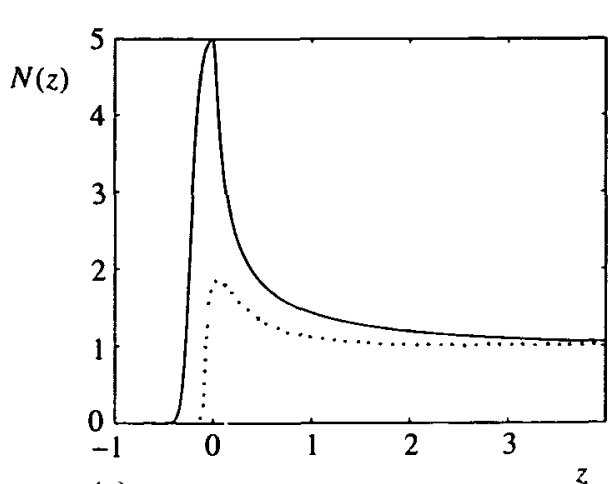

(a)

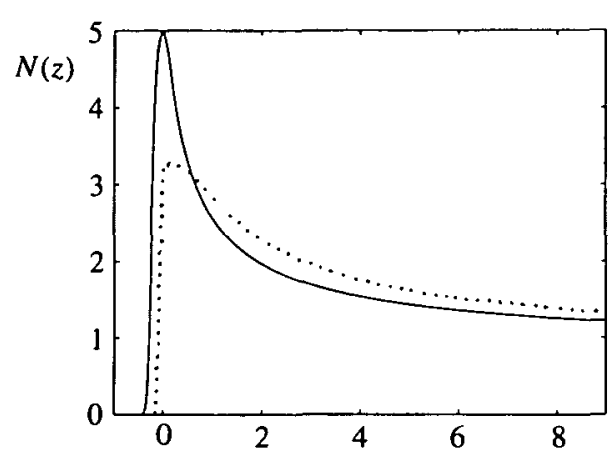

(b)

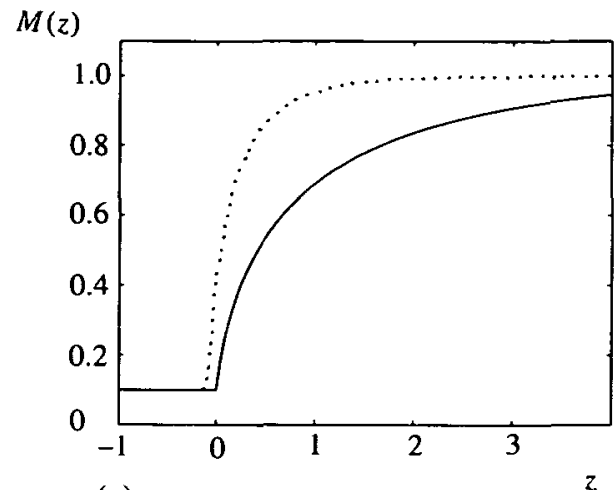

(c)

$M(z)$

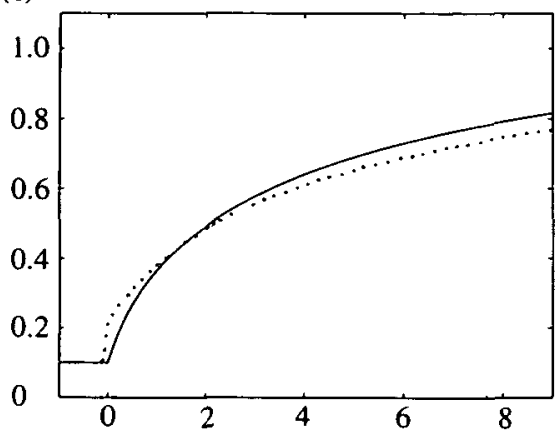

(d)

FIG. 3. Plots of the wave profiles: (a) $N(z)$ with $\epsilon=0.5$, (b) $N(z)$ with $\epsilon=0.1$, (c) $M(z)$ with $\epsilon=0.5$, and (d) $M(z)$ with $\epsilon=0.1$. Here $N$ and $M$ denote the waveforms for endothelial cells and ECM; $z$ is the travelling-wave coordinate. The parameter $\epsilon$ reflects the rate of ECM kinetics, and is thus small. (-) The lowestorder approximations given by (10) and (11) and ( . .) numerical solutions of equations (3) and (4) similar to those given in Fig. 1. This figure shows that, even to leading order, the approximate solutions determined by our perturbation methods capture the qualitative form of the solution. Higher-order correction terms are required to obtain a better quantitative fit.

4.2.3 Lowest-order matching. Denoting the lowest-order wavefront approximations by $N^{\mathrm{f}}(z)$ and $M^{\mathrm{f}}(z)$, and similarly, $N^{\mathrm{b}}(\xi)$ and $M^{\mathrm{b}}(\xi)$ for the waveback, we can match the two solutions at some point in the wave profile by continuity at that point; that is, $N^{\mathrm{f}}=N^{\mathrm{b}}$ and $M^{\mathrm{f}}=M^{\mathrm{b}}$. Thus,

$$
M^{\mathrm{b}}=m_{\text {init }}, \quad N^{\mathrm{b}}=\alpha\left(m_{\text {init }}\right) / \beta .
$$

Taking the matching point at $\xi=0$ yields the following implicit formula for $M^{\mathrm{b}}(\xi)$ :

$$
\kappa_{\alpha}^{2} \ln \left(\frac{M^{\mathrm{b}}(\xi)}{m_{\text {init }}}\right)-\left(\kappa_{\alpha}^{2}+1\right) \ln \left(\frac{1-M^{\mathrm{b}}(\xi)}{1-m_{\text {init }}}\right)-\left[M^{\mathrm{b}}(\xi)-m_{\text {init }}\right]=\alpha_{0} \xi / \beta
$$

The complete leading-order composite solution is the sum of the wavefront and waveback solutions minus the common parts, which are $m_{\text {init }}$ for $M$ and $\alpha\left(m_{1 \text { nit }}\right) / \beta$ for $N$, giving

$$
N(z)=N^{\mathrm{f}}(z)+\alpha\left[M^{\mathrm{b}}(\epsilon z)\right] / \beta-\alpha\left(m_{\mathrm{inil}}\right) / \beta,
$$




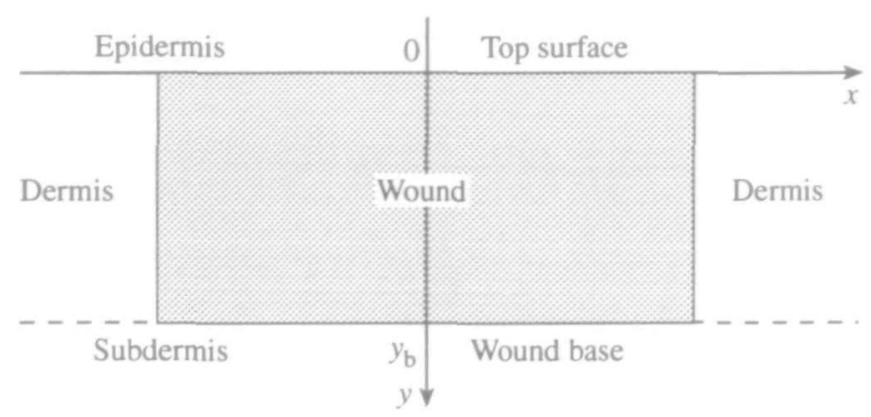

FiG. 4. A schematic cross section of the rectangular, full-thickness, dermal wound model, showing the epidermal, dermal, and subdermal layers of the skin. The dermis is defined as $-\infty<x<\infty, 0 \leqslant y \leqslant y_{b}$ and the wound space is given by $-1<x<1,0 \leqslant y \leqslant y_{b}$.

$$
M(z)=M^{\mathrm{b}}(\epsilon z)
$$

Here, $N^{\mathrm{f}}(z)$ is the solution of the Fisher equation (8) and $M^{\mathrm{b}}(\epsilon z)$ is the implicit solution of equation (9). Because the domain is contracted only in the waveback (that is, the domain transformation is one sided), this composite solution is only valid in the waveback. The wavefront solutions are simply $N^{\mathrm{f}}(z)$ and $M^{\mathrm{f}}(z)$, in the nontransformed domain.

These lowest-order approximations are compared with numerical solutions of the model equations (3) and (4) in Fig. 3, for two values of $\epsilon$. The accuracy of these approximations improves when $\epsilon$ is smaller, as expected. In addition to its role as an approximation, the perturbation approach also yields insight into the structure of the travelling-wave solutions; for example, (at least) for small $\epsilon$, the cell density in the waveback is closely related to the form of the cell proliferation term, $\alpha(m)$.

\section{Two-dimensional simulations}

So far, we have assumed that wound angiogenesis occurs in one spatial dimension, with cells and the ECM propagating towards the wound centre. In practice, however, a significant component of dermal healing proceeds from the base of the wound towards the upper surface. Subdermal tissues contain a well-developed blood-vessel network, and it is believed that a large proportion of new capillaries originate from this source during wound angiogenesis.

Here, a two-dimensional (2D) analogue of the model is formulated for wounds with a rectangular cross section, as illustrated in Fig. 4. Numerical simulations are presented below, focusing on the effects of quantitatively different boundary conditions and of wound geometries.

\subsection{The model}

The 2D versions of equations (3) and (4) are

$$
\frac{\partial n}{\partial t}+\nabla \cdot \underline{\mathcal{F}}=[\alpha(m)-\beta n] n \equiv f(n, m),
$$




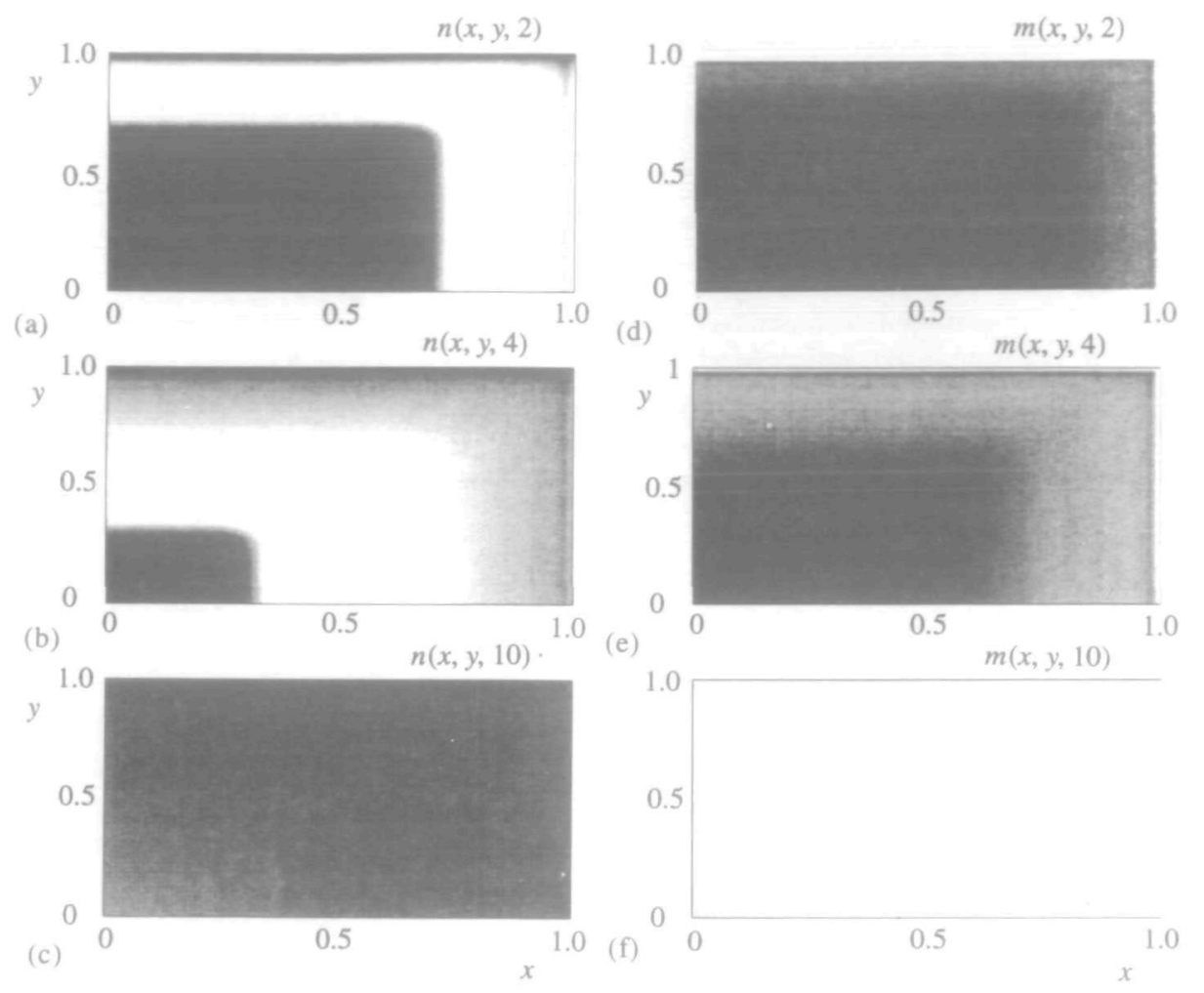

FIG. 5. Model simulations of equations 12 and 13 for wound angiogenesis in two dimensions, illustrating the propagation of $(a-c)$ endothelial cells and $(d-f)$ the ECM into the wound. Recall that $n$ and $m$ denote the density of the endothelial cells and of the ECM, respectively, while $x$ and $y$ are spatial coordinates along the surface of the skin and perpendicular to this surface, respectively. (a,d) The early stages of angiogenesis, as endothelial cells proliferate and migrate into the wound space from the wound margins at $t=2 ;(b, e)$ angiogenesis is underway, as cells invade the wound space and deposit new ECM in the waveback at $t=4$; and $(c, f)$ angiogenesis is complete, as the cell density drops from its maximum back towards normal dermal levels and the ECM gradually accumulates in the wound space at $t=10$ The end conditions are given in the text. The parameter values are given in Fig. 1(b), (d), together with $n_{b}=m_{b}=y_{b}=1$. Only the wound domain $(0 \leqslant x \leqslant 1,0 \leqslant y \leqslant 1)$ is shown here. The equations were solved numerically using the alternating-direction implicit method; this is similar in principle to the method used for 1D numerical solutions in Section 3 (see the Appendix for details). The finitedifference-approximation mesh sizes are $\Delta x=\Delta y=0.02$ and $\Delta t=0.01$. High densities are represented by lighter shading and low densities by darker shading.

$$
\frac{\partial m}{\partial t}=\epsilon(1-m) n \equiv g(n, m)
$$

where $\nabla=(\partial / \partial x, \partial / \partial y)^{\top}$ and the cell-flux vector is $\underline{\mathcal{F}}=\left(\mathcal{F}_{1}, \mathcal{F}_{2}\right)^{\top}=\chi(m) n \nabla m-$ $D(m) \nabla n$.

The boundary conditions are $n \rightarrow 1$ and $m \rightarrow 1$ as $x \rightarrow \infty$, and, by using the symmetry condition $\mathcal{F}_{1}=0$ at $x=0$, we need only consider the semi-infinite domain $x \geqslant 0$. We also assume that the cells and the ECM do not penetrate the dermis-epidermis interface, giving $\mathcal{F}_{2}=0$ at $y=0$. Finally, assuming that different biological processes occur within 
the subdermal tissue (deep wounds are beyond the scope of this paper), we stipulate the Dirichlet conditions $n=n_{\mathrm{b}}$ and $m=m_{\mathrm{b}}$ at $y=y_{\mathrm{b}}$, the wound base. The initial conditions are $n=0$ and $m=m_{\text {init }}$ in the wound space $0 \leqslant x \leqslant 1,0 \leqslant y \leqslant y_{\mathrm{b}}$, with $n=1$ and $m=1$ in the unwounded dermis $\left(x>1,0 \leqslant y \leqslant y_{b}\right)$.

\subsection{Numerical simulations}

Figure 5 shows that the numerical solutions are a direct 2D extension of those observed in 1D: waves of endothelial cells, with density $n$, and of the ECM, with density $m$, propagate in towards the wound centre; ECM accumulation follows the peak of cell proliferation until the new tissue has filled the wound and angiogenesis is complete. The similarity with the 1D-model simulations is most evident from the solution profiles along the $x=0$ and $y=0$ axes, where the system effectively mimics the ID model (due in part to the boundary conditions). The numerically observed wave speed is within a couple of per cent of the theoretical minimum, as given by the inequality in equation (7), for the simulations shown in Fig. 5. The zone of (significant) interference between the two waves moving in the $x$ - and $y$-directions lies within a narrow region situated around the line $y=x$. Here, there is a small relative increase in $n$ and a decrease in $m$ compared to the ID solutions. The quantitative dependence of the numerical solutions as the ECM kinetics parameter $\epsilon$ varies is analogous to that in $1 \mathrm{D}$.

Thus far, the subdermal cell and ECM densities have been taken as being equal to those in the dermis, which may be inaccurate. Numerical simulations reveal that away from the wound base $\left(y=y_{\mathrm{b}}\right)$ the effects of using different subdermal boundary values $\left(n_{\mathrm{b}}\right.$ and $\left.m_{\mathrm{b}}\right)$ are negligible. Near this boundary, however, there is a sharp jump in $m$ and a smooth, albeit fairly local, transition in $n$ (not shown). This is expected because cell fluxes are present in the model but the ECM has no flux; see equations (12) and (13).

Finally, we consider a quantitatively different wound geometry, in which the wound is wider than it is deep, by setting $y_{b}<1$. This is a more realistic representation of a typical excisional wound, which may be a few centimetres wide with the thickness of the skin being a few millimetres. In this case, numerical simulations of the $2 \mathrm{D}$ model show that the wave moving in the $y$-direction from the wound base reaches the wound surface before the wave moving in the $x$-direction from the adjacent dermis has reached the wound centre (not shown). These simulations also suggest that the wave speed does not depend on the depth/breadth ratio of rectangular cross-section wounds and that the two orthogonally propagating waves only interact within a narrow neighbourhood of the line $y=x$. This specific prediction of the effects of wound geometry could be directly tested by experiment.

\section{Wave speed and haptotaxis}

The travelling-wave analysis (Section 4.1) suggests that there is a minimum wave speed, $c_{\min }$, given by (7). This was derived from a linear-stability analysis of a third-order system of nonlinear ordinary differential equations given by (5) and (6) in travelling-wave phase space. Mathematically, the existence of travelling-wave solutions of the angiogenesis model is therefore a conjecture rather than a certainty; this is supported by the evidence of apparently stable numerical travelling-wave solutions of the partial differential equations (3) and (4) given in Fig. 1. In some simpler systems, stable travelling-wave solutions 


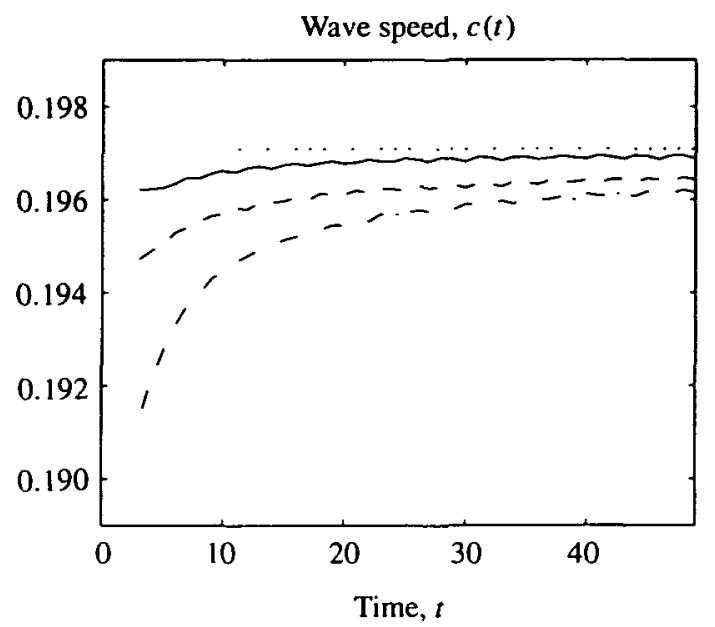

FIG. 6. The evolution of the speed $c(t)$ of angiogenesis as a function of time calculated from equations (3) and (4) (as in Fig. 1, but with the extended domain $-10 \leqslant x \leqslant 1.2$ ), for different values of the haptotaxis parameter $\chi_{0}:(-) \chi_{0}=0,(--) \chi_{0}=0.001,(-\cdots) \chi_{0}=0.005$, and $(\cdots)$ the minimum wave speed, $c_{\min } \approx 0.197$, as predicted by the travelling-wave analysis (see the text). These results illustrate that the long-term speed is essentially independent of $\chi_{0}$, but that the approach to this speed becomes slower as haptotaxis becomes stronger. Zero-flux boundary conditions are imposed at $x=-10$ with $n(x, 0)=n(0,0)$ and $m(x, 0)=m(0,0)$ for $-10 \leqslant x \leqslant 0$. The other end conditions are given in the text and the parameter values are given in the legend to Fig. 1 except $x_{0}$.

can be proven to exist and to move at the minimum speed predicted by the linear-stability analysis, with convergence towards this solution generally occurring over a long time scale (Murray, 1989; Segel, 1980).

Furthermore, the haptotactic flux term (with coefficient $\chi$ ) is nonlinear, so that the theoretical minimum wave speed, $c_{\min }$, does not account for the effects of haptotaxis. The relationships between the theoretical value $c_{\min }$ and the wave speeds observed in the numerical simulations of equations (3) and (4) are demonstrated in Fig. 6, together with the dependence of the wave speed on $\chi>0$. These results indicate that:

(i) numerical solutions evolve into travelling waves with speeds which converge towards the predicted minimum, $c_{\min }$, over a long time scale;

(ii) the effect of haptotaxis $(\chi>0)$ is to slow down the evolution towards a travelling-wave solution with speed $c_{\min }$.

Finally, if $\chi$ is sufficiently large, then instability quickly develops in the numerical simulations (not shown). This is typical of reaction-diffusion-taxis systems (Höfer et al., 1995; Jäger \& Luckhaus, 1992; Keller \& Segel, 1970; Othmer \& Stevens, 1997), and it is also expected in the numerical solutions of this model (see the Appendix).

\section{Summary}

Following a biological overview of the roles and regulators of wound-healing angiogenesis, and an outline of recent mathematical modelling work which has focused on 
soluble regulators of angiogenesis (Section 1), we developed a new continuum model for the endothelial-cell-ECM interactions involved in wound angiogenesis (Section 2).

The model consists of two coupled partial differential equations, for the endothelial-cell density and the ECM density, and it was shown that it simulated the fundamental spatiotemporal phenomena of wound angiogenesis (Section 3). In particular, travelling waves of cells and ECM propagate in towards the wound centre, with a front of proliferating endothelial cells invading the wound space ahead of ECM deposition behind the front. A minimum wave speed was deduced by linear-stability analysis which depended critically on the cell haptokinesis and proliferation terms (Section 4.1).

Numerical simulations suggest that the solution profiles exhibit a spatially elongated structure in the waveback. This observation, together with a slow-ECM-dynamics assumption, underlies the approximation analysis of the travelling-wave equations which offer analytical insight into the form of the cell and ECM wave profiles (Section 4.2).

Since many clinical dermal wounds are rectangular in cross section (including the small dead space in incisional wounds), the extension of the model to two space dimensions permitted a more realistic representation of the wound geometry and of the boundary conditions (Section 5.1). Numerical simulations of the 2D model showed that angiogenesis occurs by the cells and the ECM moving inwards from both the wound margins and the wound base, the dominant effect being determined by the ratio of the wound depth to the breadth. The properties of the angiogenic process, both parallel and perpendicular to the plane of the skin, are very similar to those for the 1D case, except within a narrow zone of interference between the orthogonally propagating waves of cells and ECM (Section 5.2).

Finally, the effects of the haptotactic flux were investigated numerically, since the travelling-wave analysis did not account for this nonlinear term. It was shown that haptotaxis transiently slows the evolution of travelling waves, as is intuitively expected in this model (Section 6).

\section{Discussion}

The primary motivation for this work and related research lies in the scope for developing practical clinical treatments for both mediating wound angiogenesis and for inhibiting tumour angiogenesis-since solid tumours cannot become malignant without angiogenesis (Folkman \& Shing, 1992; Gasparini, 1995). Recently, Chaplain and colleagues have proposed nonlinear partial-differential-equation models to delineate the mechanisms of growth-factor-driven tumour angiogenesis (Byrne \& Chaplain, 1995; Chaplain \& Stuart, $1991 \mathrm{~b}$ ). This work adopted a similar modelling framework to ours, focusing instead on the key interactions between capillary endothelial cells and their solid-state collagenous extracellular matrix substrate during wound-healing angiogenesis.

One important aspect of endothelial-cell biology that has not been explicitly addressed here is activation states. Cellular activation is an early angiogenic process in which cells are stimulated to migrate and proliferate-later, as angiogenesis ceases, the endothelial cells become quiescent and they are organized as the stable constituents of new blood capillaries (Clark, 1993; Whalen \& Zetter, 1992). The model framework presented in this work could be extended to include two cellular variables, representing active and inactive endothelial cells. Chaplain and colleagues include this feature in their models of biochemically mediated tumour angiogenesis. In our framework for ECM-mediated angiogenesis, however, 
the explicit modelling of active and quiescent cell phenotypes does not substantially affect the qualitative model predictions (the details were omitted for brevity). This is because the biological processes incorporated within the model account for ECM-induced cell activation, since cells neither proliferate nor migrate in the absence of the ECM and there is also density inhibition of these processes.

The roles of the cell-movement terms in the model, namely, the haptokinetic (unbiased) and haptotactic (biased, up ECM gradients) fluxes, are important. This model suggests that the ECM regulation of haptokinesis is the key effect, since the angiogenic wave speed (Section 4.1) and waveform (Section 4.2) depend crucially on the unbiased cell movement coefficient $D(m)$, but not on the directed motion (haptotactic) coefficient $\chi(m)$. Rather, haptotaxis effectively reduces the numerically observed wave speed by a relatively small amount, and then only transiently (Section 6). Although there is no in vivo evidence that haptotaxis specifically facilitates wound-healing angiogenesis, it is perhaps counter-intuitive that haptotaxis should elicit cell movement away from the wound centre, thereby decelerating the angiogenic process. This model prediction could be tested by a suitable in vitro study. An appropriate framework has in fact already been developed by Ingber \& Folkman (1989b), although it was actually used to study cytokine control of angiogenesis. The basic procedure is to absorb ECM proteins into plastic dishes, and to plate capillary endothelial cells onto these substrata. This technique could easily be amended to give initial configurations generating waves of endothelial-cell density. This would lead to a greater understanding of the primary mechanisms of cellular migration into healing wounds. The long-term goal of such research is of course the improved treatment of clinical wounds.

The model framework proposed in this paper may be enhanced to include a haptotaxisdriven endothelial-cell invasive process. Specifically, we have neglected the fibrin/fibronectin-rich provisional matrix, which is prominent during the early stages of wound-healing angiogenesis (McDonald, 1988; Peacock, 1984). Fibronectin is an adhesive protein which may be an important mediator of cellular migration into the wound site (Kurkinen et al., 1980; McCarthy et al., 1988; Repesh et al., 1982; Herrick et al., 1993), and its inclusion in an enlarged model may provide further insights into the process of vascular ingrowth. Another natural extension of the model would be to address the structure of the vasculature laid down by wound angiogenesis. Under optimal conditions, tight-skin species (man and pigs) create a remarkably ordered vascular stucture (Arnold et al., 1995). A number of research groups are currently developing methods for modelling orientation within connective tissue, and incorporating these methods into our model would enable mechanisms of vascular order (and its control) to be predicted. Finally, we should emphasise that cytokine control is undoubtedly a vital regulatory process in angiogenesis, which we have deliberately excluded in order to focus on ECM interactions. A comprehensive model would have to include cytokine regulation, building on the considerable existing experimental data (Ingber \& Folkman, 1989b; Le \& Bertolami, 1997).

Such extensions provide a route to the development of detailed models focusing on specific aspects of wound angiogenesis. However, even the simple model we have presented has important biological implications, and a number of experimentally testable predictions have been made which have direct clinical relevance. 


\section{Acknowledgements}

This work was supported in part by the following grants: a graduate studentship from the EPSRC (LO), EPSRC grant GR/K71394, the London Mathematical Society (Scheme 3).

\section{Appendix: Numerical-solution methods}

Here, we briefly discuss the methods used to obtain numerical solutions of the angiogenesis model, both in one and two spatial dimensions, using a semi-implicit finite-difference scheme in both cases.

\section{A.1 Crank-Nicholson method (ID)}

We require the solutions $n(x, t)$ and $m(x, t)$ of equations (3) and (4), a nonlinear parabolic partial differential equation coupled with a nonlinear ordinary differential equation. The method used to obtain approximations to these solutions is based on the popular CrankNicholson semi-implicit finite-difference scheme (Crank \& Nicholson, 1947), which uses central difference approximations for the spatial derivatives and both forward (explicit) and backward (implicit) differences (in equal proportions) for the temporal derivatives (for further details see Morton \& Mayers, 1994, Ch. 2).

We use uniform spatial and temporal grids, and denote by $n_{i}^{k}$ and $m_{i}^{k}$ the solutions at space point $x_{i}$ and time step $t_{k}$. Equation (4) for $m$ is discretized explicitly:

$$
m_{i}^{k+1}=m_{i}^{k}+(\Delta t) g\left(n_{i}^{k}, m_{i}^{k}\right) .
$$

For equation (3), central difference formulae are applied (at each time step) to the haptokinetic (unbiased) flux term:

$$
\frac{\partial}{\partial x}\left(D(m) \frac{\partial n}{\partial x}\right)\left(x_{i}, \cdot\right) \approx \frac{D\left(m_{i+\frac{1}{2}}\right)\left(n_{i+1}-n_{i}\right)-D\left(m_{i-\frac{1}{2}}\right)\left(n_{i}-n_{i-1}\right)}{(\Delta x)^{2}},
$$

where $m_{i+\frac{1}{2}}=\frac{1}{2}\left(m_{i}+m_{i+1}\right)$ etc. Similarly, for the haptotactic (biased) flux term:

$$
\frac{\partial}{\partial x}\left(\chi(m) n \frac{\partial m}{\partial x}\right)\left(x_{i}, \cdot\right) \approx \frac{\chi\left(m_{i+\frac{1}{2}}\right) n_{t+\frac{1}{2}}\left(m_{i+1}-m_{i}\right)-\chi\left(m_{i-\frac{1}{2}}\right) n_{i-\frac{1}{2}}\left(m_{i}-m_{i-1}\right)}{(\Delta x)^{2}} .
$$

As discussed above, our semi-implicit method uses the above approximations to the spatial derivatives at times $t_{k}$ and $t_{k+1}$ in equal proportions. The cell kinetics, $f$, are evaluated wholly explicitly, to avoid generating nonlinear equations for $n_{i}^{k+1}$.

A standard iterative elimination method (the Thomas algorithm) is used to solve the resulting tridiagonal system of difference equations for $n_{i}$ at each time step, subject to $n=1$ at the final mesh point, and zero-flux boundary conditions at $i=0$ (see Morton \& Mayers, 1994, for further details). If $\chi(m) \equiv 0$, the Thomas algorithm is always stable, but, more generally, stability imposes the following conditions (the derivations are omitted for brevity):

$$
\Delta x<\min _{(x, t)}\left\{\frac{2 D(m)}{\chi(m)|\partial m / \partial x|}\right\}, \quad \Delta t<\min _{(x . t)}\left\{\frac{2}{|\partial / \partial x[\chi(m) \partial m / \partial x]|}\right\} .
$$


The second of these two conditions is required only if $(\partial / \partial x)[\chi(m) \partial m / \partial x]<0$ anywhere in the domain.

These stability conditions reinforce the intuitively expected and commonly observed phenomenon that the ratio of directional to random-movement coefficients, that is, $\chi / D$, is crucial to the stability of numerical schemes for equations for the form (3). Intuitively, such instability arises because the form of the equation changes from predominantly parabolic to predominantly hyperbolic. It should also be noted that directed-motion terms can result in finite-time 'blow-up' in parabolic partial differential equations (see, for example, Jäger \& Luckhaus, 1992). Thus blow-up can potentially arise from the differential equation itself, as well as being an artefact of the numerical discretization.

\section{A.2 ADI method (2D)}

We extend our numerical scheme to two spatial dimensions using the alternating-direction implicit (ADI) method. Thus, we discretize the domain in both the $x$ - and the $y$-directions and in time using uniform grids, and divide each time step into two halves. In the first half, the spatial derivatives are evaluating explicitly in time in the $x$-direction and implicitly in the $y$-direction, and in the second half of the time step these directions are reversed. This standard technique preserves the increased stability from a semi-implicit method, while also retaining a tridiagonal system of linear equations to be solved at each time step (for further details of ADI methods, see Morton \& Mayers, 1994, Ch. 3).

\section{REFERENCES}

Alberts, B., Bray, D., Lewis, J., RAFF, M., RoberTs, K., \& WATSON, J. D., 1989. The Molecular Biology of the Cell. New York: Garland.

ARNOLD, F., \& WEST, D. C., 1992. Angiogenesis in wound healing. Pharmac. Ther. 52, 407-22.

ARnold, F., Cherry, G. W., RYan, T. J., \& ZeEman, E. C., 1995. Order and disorder in the temporal organ of wound repair. Wound Repair Regen. 3, 82.

BYRNE, H. M., \& ChAPLAIN, M. A. J., 1995. Mathematical models for tumour angiogenesisNumerical simulations and nonlinear wave analysis. Bull. Math. Biol. 57, 461-86.

ChAPLAIN, M. A. J., \& B YRNE, H. M., 1996. Mathematical modelling of wound healing and tumour growth-2 sides of the same coin. Wounds: Compend. Clin. Res. Pract., 8, 42-8.

ChaPlain, M. A. J., \& STUART, A. M., 1991a. A mathematical model for the diffusion of tumour angiogenesis factor into the surrounding host tissue. IMA J. Math. Appl. Med. Biol. 8, 191-220.

Chaplain, M. A. J., \& StuarT, A. M., 1991b. A model mechanism for the chemotactic response of endothelial cells to tumour angiogenesis factor. IMA J. Math. Appl. Med. Biol. 8, 191-220.

CLARK, R. A. F., 1988. Overview and general considerations of wound repair. In: The Molecular and Cellular Biology of Wound Repair (R. A. F. Clark \& P. M. Henson, eds.), Ch. 1, pp. 3-34. New York: Plenum.

CLARK, R. A. F., 1993. Biology of dermal wound repair. Dermatol. Clin. 11, 647-66.

CrANK, J., \& Nicholson, P., 1947. A practical method for numerical evaluation of solutions of partial differential equations of the heat-conduction type. Proc. Camb. Philos. Soc. 43, 50-67.

DALE, P. D., MAINI, P. K., \& SHERRATT, J. A., 1994. Mathematical modelling of corneal epithelial wound healing. Math. Biosci. 124, 127-47.

Dickinson, R. B., \& TRANQUiLLo, R. T., 1993. A stochastic model for adhesion-mediated cell random motility and haptokinesis. J. Math. Biol. 31, 563-600.

Drucker, W., Pearce, F., Glassheidenreich, L., hopf, H., Powell, C., Ochsner, M. G., Frankel, H., Murray, D., Nelson, M., Champion, H., RozyCKI, G., Silva, J., Malcolm, D., Denobile, J., Harviel, D., Rich, N., \& Hunt, T., 1996. Subcutaneous tissue 
oxygen pressure-A reliable index of peripheral perfusion in humans after injury. J. Trauma 40, S116-S122.

Dyson, M., Young, S. R., HART, J., LYNCH, J. A., \& LANG, S., 1992. Comparison of the effects of moist and dry conditions on the process of angiogenesis during dermal repair. J. Invest. Dermatol. 99, 729-33.

EDELSTEIN-KESHET, L., 1988. Mathematical Models in Biology. New York: Random House.

FISHER, R. A., 1937. The wave of advance of advantageous genes. Ann. Eugenics 7, 353-69.

Folkman, J., \& ShING, Y., 1992. Angiogenesis. J. Biol. Chem. 267, 10931-4.

GASPARINI, G., 1995. Prognostic and predictive value of the determination of tumour angiogenesis in primary solid tumours. Eur. J. Cancer A 31, 24.

Herrick, S. E., Ireland, G. W., Sloan, P., MCCollum, C. N., \& Ferguson, M. W. J., 1993. Sequential changes in histological pattern and extracellular matrix during the healing of chronic venous leg ulcers. J. Cell. Biochem S17E, 120.

HöFER, T., SHERRATT, J. A., \& MAINI, P. K., 1995. Cellular pattern formation during dictyostelium aggregation. Physica D, 85, 424-44.

INGBER, D., \& FOLKMAN, J., 1988. Inhibition of angiogenesis through modulation of collagen metabolism. Lab. Invest. 59, 44-51.

INGBER, D. E., 1993. Cellular tensegrity: Defining new rules of biological design that govern the cytoskeleton. J. Cell Sci. 104, 613-27.

INGBER, D. E., \& FolkmAN, J., 1989a. How does the ECM control capillary morphogenesis? Cell $58,803-5$

INGBER, D. E., \& FOLKMAN, J., 1989b. Mechanochemical switching between growth and differentiation during fibroblast growth factor stimulated angiogenesis in vitro-Role of extracellular matrix. J. Cell Biol. 109, 317-30.

IRUELA-ARISPE, M. L., \& SAGE, E. H., 1993. Endothelial cells exhibiting angiogenesis in vitro proliferate in response to TGF $\beta 1$. J. Cell Biochem. 52, 414-30.

JÄGER, W., \& LUCKHAUS, S., 1992. On explosions of solutions to a system of partial differential equations modelling chemotaxis. Trans. Am. Math. Soc. 329, 819-24.

Jonsson, K., Jensen, J. A., Goodson, W. H., Scheuenstuhl, H., West, J., HoPf, H. W., \& HUNT, T., 1991. Tissue oxygenation, anemia, and perfusion in relation to wound healing in surgical patients. Ann. Surg. 214,605-13.

KELLER, E. F., \& SEGEL, L. A., 1970. The initiation of slime mold aggregation viewed as an instability. J. Theor. Biol. 26, 399-415.

KNighton, D. R., HunT, T. K., Thakrai, K. K., \& GoOdSON, W. H., 1982. Role of platelets and fibrin in the healing sequence-An in vivo study of angiogenesis and collagen synthesis. Ann. Surg. 196, 379-88.

Kurkinen, M., VAheri, A., Roberts, P. J., \& Stenman, S., 1980. Sequential appearance of fibronectin and collagen in experimental granulation tissue. Lab. Invest. 43, 47-51.

LE, A. \& BERTOLAMI, C., 1997. Regulation of dermal wound angiogenesis-Role of vEGF. $J$. Dental Res. 76, 3151.

MADRI, J. A., \& PRATT, B. M., 1988. Angiogenesis. In: The Molecular and Cellular Biology of Wound Repair (R. A. F. Clark \& P. M. Henson, eds.), Ch. 15, pp. 337-58. New York: Plenum.

MAST, B. A. 1992. The skin. In: Wound Healing: Biochemical and Clinical Aspects (I. K. Cohen, R. F. Diegelmann, \& W. J. Linblad, eds.), Ch. 22, pp. 344-55. Philadelphia, PA: Saunders.

MCCARTHY, J. B., SAS, D. F., \& FURCHT, L. T., 1988. Mechanisms of parenchymal cell migration into wounds. In: The Molecular and Cellular Biology of Wound Repair (R. A. F. Clark \& P. M. Henson, eds.), Ch. 13, pp. 281-319. New York: Plenum.

MCDONALD, J. A, 1988. Fibronectin: A primitive matrix. In: The Molecular and Cellular Biology of Wound Repair (R. A. F. Clark \& P. M. Henson, eds.), Ch. 18, pp. 405-35. New York: Plenum.

MORTON, K. W. \& MAYERS, D. F., 1994. Numerical Solution of Partial Differential Equations. Cambridge University Press.

MurRaY, J. D., 1989. Mathematical Biology: New York: Springer-Verlag.

Nusgens, B., Merill, C., LAPIERE, C., \& Bell, E., 1984. Collagen biosynthesis by cells in a tissue equivalent matrix in vitro. Collagen Rel. Res. 4, 351-63.

Olsen, L., SherRatT, J. A., \& MaINI, P. K., 1995. A mechanochemical model for adult dermal 
wound contraction and the permanence of the contracted tissue displacement profile. $J$. Theor. Biol. 177, 113-28.

Olsen, L., SherRatT, J. A., \& Maini, P. K., 1996. A mathematical model for fibro-proliferative wound healing disorders. Bull. Math. Biol. 58, 787-808.

OTHMER, H. G., \& STEVENS, A., 1997. Aggregation, blowup and collapse: The ABCs of taxis in reinforced random walks. Submitted.

PEACOCK, E. E., 1984. Wound Repair. Philadelphia, PA: Saunders.

PetTet, G. J., Byrne, H. M., MCElwain, D. L. S., \& Norbury, J., 1996. A model of woundhealing angiogenesis in soft tissue. Math Biosci. 136, 35-63.

REPESH, L. A., FITZGERALD, T. J., \& FURCHT, L. T., 1982. Fibronectin involvement in granulation tissue and wound healing in rabbits. J. Histochem. Cytochem. 30, 351-8.

RYAN, T. J., 1989. Biochemical consequences of mechanical forces generated by distension and distortion. J. Am. Acad. Dermatol. 21, 115-30.

Sato, N., Beitz, J. G., Kato, J., Yamamoto, M., Clark, J. W., Calabresi, P., \& A. R. FRACKELTON, J., 1993. Platelet-derived growth factor indirectly stimulates angiogenesis in vitro. Am. J. Pathol. 142, 1119-30.

SEGEL, L. A., 1980. Mathematical Models in Molecular and Cellular Biology. Cambridge University Press.

ShERRATT, J. A., 1994. Chemotaxis and chemokinesis in eukaryotic cells: The Keller-Segel equations as an approximation to a detailed model. Bull. Math. Biol. 56, 129-46.

Sherratt, J. A., Martin, P., Murray, J. D., \& LEWIS, J., 1992. Mathematical models of wound healing in embryonic and adult epidermis. IMA J. Math. Appl. Med. Biol. 9, 177-96.

STOPAK, D., \& HARRIS, A. K., 1982. Connective tissue morphogenesis by fibroblast traction. I: Tissue culture observations. Dev. Biol. 90, 383-98.

VERNON; R. B., \& SAGE, E. H., 1995. Between molecules and morphology. Extracellular matrix and creation of vascular form. Am. J. Pathol. 147, 873-83.

WEINBERG, C. B., \& BELL, E., 1985. Regulation of proliferation of bovine aortic endothelial cells, smooth muscle cells and adventitial fibroblasts in collagen lattices. J. Cell. Physiol. 122, 41014.

Whalen, G. F., \& ZeTter, B. R., 1992. Angiogenesis. In: Wound Healing: Biochemical and Clinical Aspects (I. K. Cohen, R. F. Diegelmann, \& W. J. Lindblad, eds.), Ch. 5, pp. 77-95. Philadelphia, PA: Saunders.

YoshizATO, K., TAIRA, T., \& YAMAMOTO, N., 1985. Growth inhibition of human fibroblasts by reconstituted collagen fibrils. Biomed. Res. 6, 61-71. 